\title{
Urinary Steroid Assays and Response to Endocrine Ablation
}

\author{
B. S. THOMAS,* M.I.BIOL.; R. D. BULBROOK,* M.SC., PH.D. ; J. L. HAYWARD, $†$ M.B., B.S., F.R.c.s.
}

Brit. med. F., 1967, 3, 523-524

Bulbrook, Greenwood, and Hayward (1960) found a close relation between the subsequent clinical response of patients with advanced breast cancer to endocrine ablation and the amounts of androgen and corticosteroid metabolites in the urine before operation. They noted that in their small series of patients the best correlation between steroid assay results and clinical response was obtained if a discriminant function was used. This function was: $80-80$ (17-OHCS $\mathrm{mg}$./24 hours) + aetiocholanalone ( $\mu \mathrm{g} . / 24$ hours). The function is positive in patients excreting large amounts of aetiocholanolone relative to the amounts of $17-\mathrm{OHCS}$, and patients in this category generally responded well to endocrine ablation. Conversely, patients in whom the discriminant is negative (low aetiocholanolone excretion relative to that of the 17-OHCS) generally do not benefit from adrenalectomy or hypophysectomy (see Hayward and Bulbrook, 1965).

Miller, Durant, Jacobs, and Allison (1967) have described a high degree of correlation between the discriminant and a simple ratio of the total urinary 11-deoxy-17-oxosteroids and the 17-OHCS. It should follow, therefore, that their ratio would be as useful as the discriminant in the prediction of response to adrenalectomy or hypophysectomy.

We had available the assay results of the total 11-deoxy-17oxosteroids, the 17-OHCS, and the discriminant in 210 patients with advanced breast cancer whose clinical response to endocrine ablation was already known. This paper is a report of a retrospective comparison of the predictive value of the discriminant and of the ratio described by Miller et al. (1967).

\section{Methods}

The 17-OHCS were determined by the method of Appleby, Gibson, Norymberski, and Stubbs (1955). It should be noted that Miller et al. (1967) used the method of Metcalf (1963) for the determination of the 17-OHCS. These methods are not strictly comparable: the latter method is based on a shortterm hot oxidation of the 17-OHCS with sodium metaperiodate and the results are expressed in cortisol equivalents. The method of Appleby et al. (1955) is based on oxidation at room temperature with sodium bismuthate and results are expressed in terms of dehydroepiandrosterone. In practice, this means that the results of the 17-OHCS assays may differ by $1-2 \mathrm{mg}$./ 24 hours, but the difference between the methods should be approximately constant (see, for example, Thomas, 1965).

In the first 119 patients dehydroepiandrosterone, androsterone, and aetiocholanolone were determined by the method of Kellie and Wade (1957) and the value for the total 11deoxy-17-oxosteroids was obtained by summing the amounts of these three steroids. In the remaining patients the total 11deoxy-17-oxosteroids were measured as a group by the method of Thomas and Bulbrook (1964). The results of the two methods are very similar.

Either three or five consecutive 24-hour urine specimens were collected from 210 patients with metastatic breast cancer awaiting combined oophorectomy and adrenalectomy, or hypophysec-

\footnotetext{
* Division of Chemistry and Biochemistry, Imperial Cancer Research Fund, London W.C.2.

t Department of Surgery, Guy's Hospital, London S.E.1.
}

tomy. Clinical evaluation of response to treatment has been described in detail by Hayward and Bulbrook (1965) and the methods used have been discussed at length (see Hayward and Bulbrook, 1966).

\section{Results}

The usefulness of the discriminant function in differentiating between responsive and unresponsive patients at adrenalectomy or hypophysectomy can be judged from the data in the Table. In this series of 210 patients the remission rate is three times as great in patients with positive discriminants as in those with negative discriminants.

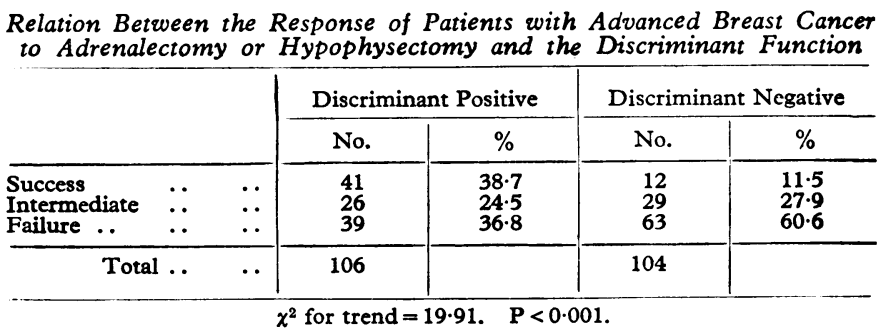

The relation between the clinical response of patients to adrenalectomy or hypophysectomy and either the discriminant function or a ratio of the total 11-deoxy-17-oxosteroids to the 17-OHCS is shown in Fig. 1. It can be seen that the majority of successful responses to treatment occur in patients with positive discriminants and with high ratios. Conversely, the

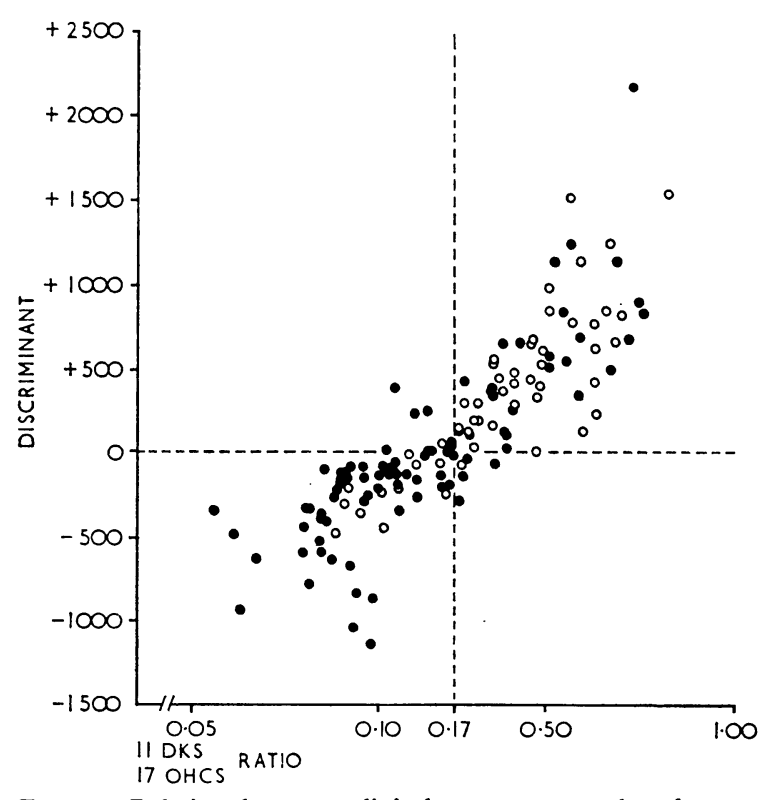

Fig. 1.-Relation between clinical response to adrenalectomy FIG. 1.-Relation between clinical response to adrenalectomy or hypophysectomy and the discriminant function or the 11deoxy-17-oxosteroid/17-OHCS ratio. This Figure is based on results from 197 patients, of whom 52 responded to treatment
and 92 failed to respond. $\mathrm{O}=$ Successful response. Failure. 
majority of the failures occur in patients with negative discriminants and low ratios. If a ratio is used such that

$$
\frac{11 \text {-deoxy-17-oxosteroids (mg./24 hours) }}{17-\text { OHCS (mg./24 hours) }}=0.17
$$

then patients with ratios equal to or below this figure have a response rate that is identical to that found for patients with negative discriminants. Similarly, patients with a ratio above 0.17 also have a response rate that is virtually the same as that for patients with positive discriminants (see Fig. 2).

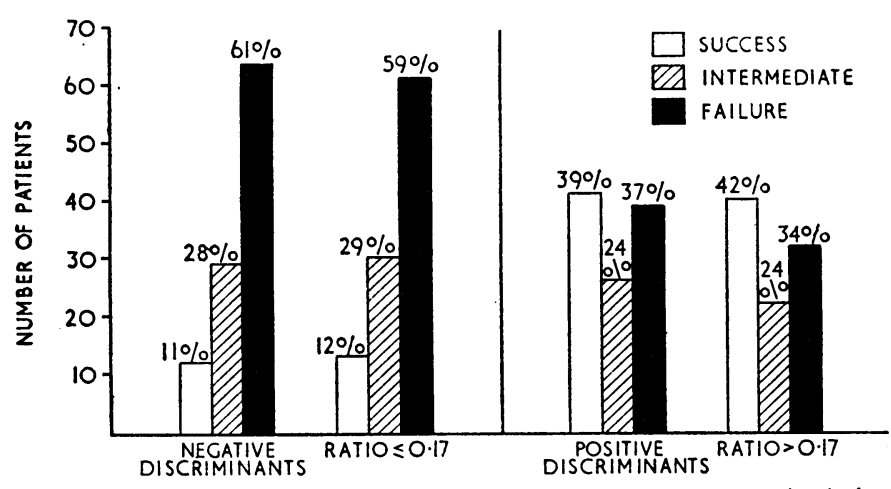

FIG. 2-Clinical response to endocrine ablation in relation to discriminant or ratio of 11 -deoxy-17-oxosteroids to the $17-$ OHCS.

\section{Discussion}

The results in this paper show that a simple ratio of the 11-deoxy-17-oxosteroids to the 17-OHCS can be chosen which in retrospect will give the same prediction of response to endocrine ablation as the discriminant function. This finding is to be expected from the results of Miller et al. (1967). It should be recalled that the discriminant function was calculated originally from the results of an investigation of a small series of 59 patients, of whom only 14 responded to treatment. The misclassification of only one or two of these successful cases would have had a pronounced effect, and a discriminant function gave the best separation of responsive and unresponsive cases at that time.

The easier analytical methods used by Miller et al. (1967) could be substituted, therefore, for the more cumbersome ones required for the calculation of the original discriminant for patients with advanced breast cancer.

There is a considerable degree of misclassification of response, whether the discriminant or the ratio is used. The results in the Table show that $37 \%$ of patients with positive discriminants fail to respond to treatment, whereas $12 \%$ of patients with negative discriminants enjoy an objective remission of the disease. To a very large extent the same patients are misclassified by the ratio or by the discriminant.

While there is now a considerable consensus of agreement that the response of patients to adrenalectomy or hypophysectomy is related to the amounts of androgen and corticosteroid metabolites found in the urine before treatment, the results of individual workers differ in detail and emphasis. Thus, Juret, Hayem, and Flaisler (1964) found that the sum of the androsterone and aetiocholanolone values was related to the response of their patients to destruction of the pituitary by yttrium-90.

Wilson, Crocker, Fairgrieve, Bartholomay, Emerson, and Moore (1967) have found a relation between clinical response to adrenalectomy and a discriminant function based on 17-oxosteroid and 17-OHCS response to A.C.T.H. stimulation, and they also make use of the predictive information provided by the free period (see below). Kumaoka, Abe, Sakauchi, Takatana, and Kusama (1966) have found significant differences in total 17-oxosteroid excretion between their responsive and unresponsive patients.

An additional complication is the recognition that factors other than the endocrine environment are of considerable importance in relation to response to treatment. The free period (the time between mastectomy and first recurrence of the disease) is longer in responsive patients than in unresponsive patients (see Taylor, 1966). When the length of the free period is taken into account in conjunction with the steroid assay results, there is a considerable increase in the accuracy of prediction of response. The menopausal status of the patient is also important (see Bulbrook, 1966). Whether the ratio described by Miller et al. (1967) will be as useful as the discriminant when employed in conjunction with such factors in patients with advanced breast cancer has yet to be determined.

The most probable development in the future is likely to be the development of even more complicated methods of discriminating between responsive and unresponsive patients as the importance of steroids other than the androgens and corticoids is recognized and as the effect of biological variables such as free period and menopausal status are appreciated.

Bulbrook, Hayward, and Thomas (1964) found that the discriminant function was related to recurrence and death rate after mastectomy. The use of a simple ratio instead of the discriminant in these patients is now being evaluated.

\section{Summary}

In women with breast cancer there is a high degree of correlation between the urinary discriminant function 80-80 (17-OHCS mg./24 hours) + aetiocholanolone ( $\mu \mathrm{g} . / 24$ hours) and a simple ratio of total 11-deoxy-17-oxosteroids to the 17-OHCS.

In a retrospective study on 210 women with advanced breast cancer subjected to either adrenalectomy or hypophysectomy the prognostic values of both the discriminant function and the simplified ratio were virtually identical.

Easier analytical methods are used for the calculation of the 11-deoxy-17-oxosteroid:17-OHCS ratio than for the calculation of the discriminant function.

\section{REFERENCES}

Appleby, J. I., Gibson, G., Norymberski, J. K., and Stubbs, R. D. (1955). Biochem. F., 60, 453

Bulbrook, R. D. (1966). In Clinical Evaluation in Breast Cancer, edited by J. L. Hayward and R. D. Bulbrook, p. 77. London.

Greenwood, F. C., and Hayward, J. L. (1960). Lancet, $1,1154$.

- Hayward, J. L., and Thomas, B. S. (1964). Ibid., 1, 945

Hayward J L and Bulbrok R D. (1965) Cancer Res. $25,1129$.

Hayward, (editors) (1966). Clinical Evaluation in Breast Cancer. London.

Juret, P., Hayem, M., and Flaisler, A. (1964). 7. Chir. (Paris), 87, 409.

Kellie, A. E., and Wade, A. P. (1957). Biochem. F., 66, 196.

Kumaoka, S., Abe, A., Sakauchi, N.. Takatani, O., and Kusama, M. (1966). Abstracts 9th Int. Cancer Congress, Tokyo, p. 653.

Metcalf, M. G. (1963). 7. Endocr., 26, 415.

Miller, H., Durant, J. A., Jacobs, A. G., and Allison, J. F. (1967). Brit. med. $\mathcal{F} ., 1,147$.

Taylor, S. G. (1966). In Clinical Evaluation in Breast Cancer, edited by J. L. Hayward and R. D. Bulbrook, p. 205. London.

Thomas, B. S. (1965). \%. clin. Endocr., 25, 710.

Thomas, B. Bulbrook, R. D. (1964). F. Chromat., 14, 28.

Wilson, R. E., Crocker, D. W., Fairgrieve, J., Bartholomay, A. F. Emerson, K., and Moore, F. D. (1967).' F." Amer. med. Ass., 199, 474. 